\title{
Ist die Vitaminsubstitution bei Epilepsie-Patienten sinnvoll?
}

Rund zwei Drittel aller Epilepsie-Patienten bedürfen einer lebenslangen Anfallsprophylaxe - mit allen potenziellen Risiken einer solchen Dauertherapie. Beispielsweise können Antikonvulsiva mit Stoffwechselvorgängen interagieren, die bei der Aufnahme und Verwertung von Vitaminen eine Rolle spielen.

Q ereits Ende der 1970er-Jahre wurde D über die „Osteopathia antiepileptica“ bei Patienten unter Langzeittherapie mit Phenytoin berichtet [1]. Als einer der wesentlichen Störfaktoren wurde der gesteigerte Vitamin D3-Abbau durch enzyminduzierende Antikonvulsiva wie Carbamazepin, Phenytoin oder Phenobarbital identifiziert. Als ganz aktuellen - gewissermaßen umgekehrten - Beweis zitierte Professor Christian Elger, Bonn, eine Studie, in der zwei Jahre nach Absetzen von Phenytoin beziehungsweise Umstellung auf Levetiracetam die Knochendichte wieder signifikant zugenommen hatte [2]. Es ist aber wohl nicht allein die Aktivierung des CYP-450-Systems, das den Vitamin-D3-Abbau beschleunigt, denn die Knochenstabilität wird auch durch Valproat, einen Enzymininhibitor, verringert.

Der häufigste Vitaminmangel in westlichen Industrieländern betrifft die Folsäure (Vitamin B9). Sie gehört zusammen mit den Vitaminen B6 und B12 zu wichtigen Kofaktoren im Methionin-Homozysteinstoffwechsel. Der vor allem durch enzyminduzierende Antikonvulsiva induzierte Mangel an Folsäure und Vitamin B12 kann zur Erhöhung der Homozysteinkonzentration führen, was mit einem beschleunigten kognitiven Abbau und einer größeren Wahrscheinlichkeit, an der Alzheimer-Demenz zu erkranken, verbunden ist. Der Prozess lässt sich aber umkehren, denn unter der Substitution von Folsäure und Vitamin-B12 fällt der Homozysteinspiegel wieder und bei Menschen mit milder kognitiver Einschränkung wird das Fortschreiten der Hirnatrophie verlangsamt [3].

Eine spezielle Rolle spielt Valproat. Obwohl es nicht wie die anderen Antikonvulsiva enzyminduzierend wirkt und die Vitamin-B12-Serumspiegel nicht absinken, sondern ansteigen lässt, erhöht es die Homozysteinkonzentration [4].
Wahrscheinlich induziert Valproat den intrazellularen Vitamin-B12-Mangel über einen anderen Mechanismus, vermutet Elger. Die Konsequenzen ließen sich an der reduzierten Kognitionsleistung von Kindern ablesen, deren Epilepsie-kranke Mütter in der Schwangerschaft Valproat eingenommen hätten [5].

Insgesamt hält Elger die Beweislage zum Einfluss der Antikonvulsiva auf die Konzentration der genannten Vitamine für recht gut und erachtet aufgrund der erheblichen Langzeitkonsequenzen bei Epilepsie-Kranken eine Substition für sinnvoll.

Gabriele Blaeser-Kiel

1. Mehregan $\mathrm{U}$ et al. Arch Psychiatr Nervenkr 1979; 226: 299-310

2. Phabphal K et al. Epilepsia 2013 [Epub ahead of print]

3. Smith AD et al. PLoS ONE 2010; 5 (9): e12244

4. Semmler A et al. Clin Chem Lab Med 2013; 51: 665-9

5. Meador KJ et al. N Engl J Med 2009; 360: 1597-1605

8. Gemeinsame Jahrestagung der Deutschen und Österreichischen Gesellschaften für Epileptologie und der Schweizerischen Liga gegen Epilepsie, Interlaken/Schweiz, 8.-11.5.2013

\section{Prädiktion von Pharmakoresistenz und Nebenwirkungsrisiko}

Derzeit arbeitet man in der Forschung verstärkt daran, genotypische Besonderheiten als Biomarker zur Vorhersage einer Pharmakoresistenz und für das Auftreten spezifischer Nebenwirkungen zu etablieren.

D as SCN1A-Gen kodiert für einen Natriumkanal (Nav1.1), der bei der Erregungsbildung und -fortleitung in inhibitorischen Neuronen eine wichtige Rolle spielt. Über diesen Kanal entfalten Natriumkanalblocker wie Lamotrigin (LTG) ihre Wirkung. Bei Epilepsie-Patienten mit mutiertem SCN1A-Gen sind diese Substanzen oft nicht wirksam; sie können sogar anfallsverstärkend wirken. Die Mutation bewirkt einen Funktionsverlust dieses Natriumkanals; die Folgen sind Überaktivität und epileptische Anfälle. Ein Natriumkanalblocker stört in dieser Situation das bereits funktionell geschädigte System zusätzlich. Liegt das SCN1A-Gen in der Variante
rs3812718AA vor, werden höhere Dosen von Carbamazepin (CBZ) oder Phenytoin (PHT) benötigt, um therapeutisch wirksam zu sein. Auch Nebenwirkungen von Antiepileptika können genetisch bedingt sein. So geht der Genotyp CYP2C $9{ }^{*} 6$ des hepatischen Cytochrom$\mathrm{P}$-Systems mit einer verlangsamten $\mathrm{Me}$ tabolisierung und dadurch verlängerten Halbwertszeit von PHT einher.

$\mathrm{Zu}$ den schweren arzneimittelbedingten Reaktionen gehören Hypersensitivitätsreaktionen wie Steven-Johnson-Syndrom (SJS) und toxische epidermale $\mathrm{Ne}$ krolyse (TEN). Hier kommt es aufgrund einer T-Zell-vermittelten Reaktion zur Apoptose von Keratinozyten. Personen mit dem Serotyp HLA-B ${ }^{\star} 1502$, der in der asiatischen Bevölkerung häufig ist, haben unter einer Therapie mit CBZ ein erhöhtes Risiko von fast $10 \%$ für diese schweren Hypersensitivitätsreaktionen. Die „Federal Drug Administration“ (FDA) empfiehlt daher bei asiatischen Epilepsie-Patienten vor einer CBZ-Gabe den HLA-Status zu bestimmen. Auch der Serotyp HLA-A ${ }^{\star} 3101$, der bei 2-5\% aller Europäer nachweisbar ist, geht mit einem erhöhten Risiko für allergische Reaktionen wie makulopapulöses Erythem und SJS/TEN unter einer CBZTherapie einher $(26 \%$ versus $8 \%$ bei Kontrollpersonen). Dr. Katharina Arnheim

Vortrag von Professor Y. Weber bei der 8. Gemeinsamen Jahrestagung der Deutschen und Österreichischen Gesellschaften für Epileptologie und der Schweizerischen Liga gegen Epilepsie, Interlaken/Schweiz, 8. - 11.5.2013 